\title{
Parallel Volume Rendering Algorithm of Volume Mineralization Model based on GPU
}

\author{
Bin Ma, Yaohe Liu*, and Hyder Abbas \\ North China University of Water Resources and Electric Power, Zhengzhou, 450046, China
}

\begin{abstract}
Volume rendering is an important technique in data visualization, but it has some problems, such as low sampling efficiency and slow drawing speed. This is especially true for the model of body visualization calculation, where the scene rendering speed is too large and the throughput rate is too high. To improve the drawing speed of the volume rendering algorithm, a method of parallel GPU computing is proposed to optimize the algorithm. A parallel volume rendering algorithm based on GPU is implemented to improve the rendering speed, and the experimental results show that the algorithm can improve the speed without affecting the image quality.
\end{abstract}

Keywords: body mineralization; volume rendering; rendering algorithm; GPU; data visualization; parallel computation

(Submitted on May 9, 2018; Revised on June 23, 2018; Accepted on July 26, 2018)

() 2018 Totem Publisher, Inc. All rights reserved.

\section{Introduction}

The digital mine is the one in which science and technology innovation and development strategy use information technology to transform the traditional industry. The three-dimensional digital modeling of orebody is one of the key technologies of the digital mine and is the basis of realizing digital mine [1]. However, in practical engineering application, the three-dimensional modeling and visualization system of orebody, especially the three-dimensional visualization system based on the model of body mineralization, is unable to fully play to its advantages. The main reason lies in the large size of the ore-rock space, which is difficult to express. Volume rendering is a whole display of the three-dimensional data field, and the micro-structure and small changes in the data field can be displayed in different degrees.

However, the volume rendering algorithm casts a large amount of light data and requires multiple sampling for each light, resulting in slower image generation [2-3]. Therefore, many scholars at home and abroad have done a lot of research on improving volume drawing speed [4], including Fang Jun and Fang Xiaoyang [5]. By introducing collision detection technology, the method of ray jumping is used to skip the resampling of the air in the bounding box of collision detection, which accelerates the process of light synthesis [6]. An efficient interpolation method is realized by using the mathematical characteristics of parallel line cutting to cast light, which improves the speed of volume rendering [7]. An adaptive ray casting algorithm with improved intersection is proposed to determine the adaptive sampling step. The algorithm mainly applies methods of polygon scan conversion and voxelization of casting rays. The algorithm uses the method of determining the convex hull of a set and the intersecting algorithm related directly to $\mathrm{x}, \mathrm{y}, \mathrm{z}$ family planes to clip data sets and rays.

Although these algorithms improve the rendering speed of the ray casting algorithm to some extent, the images are only analyzed from the angle of the process of the Ray projectile rendering algorithm. In this paper, a volume rendering optimization algorithm based on GPU parallel computation is proposed.

\footnotetext{
* Corresponding author.

E-mail address: 1yh_950622@163.com
} 


\section{Description of System Model and GPU}

\subsection{Orebody Model}

Orebody is one of the main objects of geological engineering, and it is a kind of special geologic body.

The mining technicians not only focus on the spatial attribute of the general geological body, but also the chemical composition, the physical property and the ore-rock property. According to the different emphases of the ore body, the simulation model can be divided into three kinds, such as digital model, geometrical model and ore-mineralization model. The body mineralization model is a solid model based on the volume visualization theory, which can be used to describe the geometrical shape of the ore body and the valuation of the orebody grade and reserve by means of a mixed geometry model and digital model.

In this archetype, a three-dimensional model is constructed by the set operation of the volume element.

\subsection{GPU Parallel Programming Technology}

As far as the parallel computing technology is concerned, it mainly includes a single core multi instruction parallel, multicore parallel, multiprocessor parallel and cluster distributed parallelism.

On the basis of contrasting and analyzing these four kinds of parallel technologies, this project team completes the development of the system prototype with the CPU + GPU cooperative method. In this framework, the computational task of the body mineralization model is accomplished through efficient collaboration between the CPU and GPU. The system is divided into CPU end system and GPU end system according to the realization location. Because the CPU architecture is mainly designed for a serial code in the microcomputer system, it is suitable for logic control, visit management, sequential execution and so on. The CPU-side system is mainly responsible for data exchange between algorithms (such as reading data from the external storage into memory,), data communication, process scheduling, computing task allocation and management, logical transaction processing, etc. The GPU architecture has many ALU computing units, which is suitable for high concurrency computation, but is not suitable for frequent data exchange and excessive logic processing.

At this point, the GPU-side system mainly performs parallel computation and pipelined data processing, and realizes the parallel modeling algorithm and parallel volume rendering algorithm of the body mineralization model.

\section{Parallel Model and Parallel Rendering}

\subsection{Parallel Model in Mineralization Model}

The volume rendering technique is mainly used to simulate the entity model by drawing the body and the attributes of the entity elements. Because the Volume pixel is divided into a small spatial stereo structure with Volume pixel as the unit, for each small Volume pixel, the property can be assigned and the location of the space is precisely positioned. So, this method focuses on the representation of the inner structure of the three-dimensional space and can describe the rich properties of the object. Through the description of the volume of the three-dimensional space of the target representation, the advantage is suitable for space operation and analysis. But in the case of high accuracy of object expression, the volume will produce many Volume pixels, which will give the system simulation of large storage space. The calculation speed is also slow. At the same time, when the observation point changes or the object in the three-dimensional space coordinate transformations, it is necessary to reuse the algorithm to generate display data, which makes this method computationally large.

It is difficult to use traditional graphics hardware to realize real-time rendering. Considering that the volume rendering algorithm has the advantages of good rendering effect, easy realization and suitable for GPU acceleration, this algorithm is used to draw the body mineralization model, as shown in Figure 1. The line of sight enters the model from the injection point, leaves the model from the ejection point, and intersects the Volume pixel in the mineralization model.

In the direction of the front and back of the line of sight, it samples and synthesizes the properties of each Volume pixel (map to opacity, color, etc.) and display on the screen to complete the three-dimensional drawing of the orebody model. 


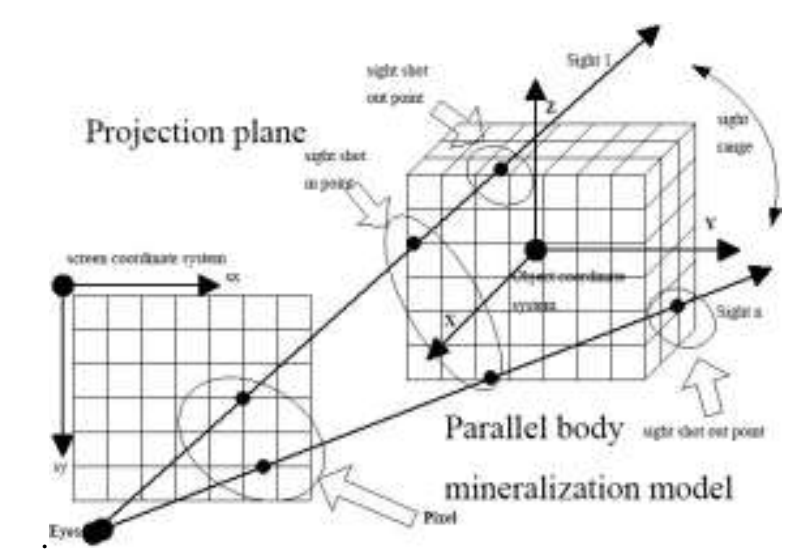

Figure 1. Volume rendering algorithm for parallel body mineralization model

\subsection{Parallel Rendering}

In this paper, the volume rendering algorithm of the analytic body mineralized mold is drawn, and each line of sight passes through a number of the Volume pixel in the body-element set and reaches the outside of the volume pixel collection. According to the line of sight, it forms a volume pixel chain according to Equation (1) and Equation (2) resampling, and synthesis, as shown in Figure 2. But, for the volume rendering algorithm in the process of image generation, each line of sight must pass through the orebody Volume pixel set, which leads to its slow drawing speed. From Figure 2, it is found that not only is the Volume pixel information of the body mineralization model independent, but the volume rendering algorithm is also relatively independent in the projection of line of sight and the synthetic data process, so the volume rendering algorithm is suitable for parallel processing.

$$
\begin{gathered}
C_{\text {out }}=C_{\text {in }} a_{\text {in }}+C_{\text {now }} a_{\text {now }}\left(1-a_{\text {in }}\right) \\
a_{\text {out }}=a_{i n}+a_{\text {now }}\left(1-a_{i n}\right)
\end{gathered}
$$

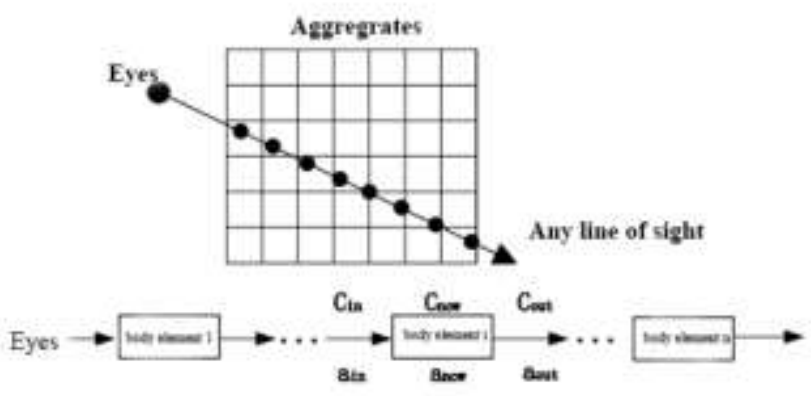

Figure 2. Parallel rendering view sketch

\section{Algorithm Implementation and Optimization}

\subsection{Algorithm Implementation}

The parallel volume rendering algorithm based on the model of body mineralization draws a three-dimensional orebody model with multiple rays at the same time from the point of view through the screen to reach the body aggregates, and in the direction of the line of sight in parallel to sample and synthesize the orebody grade value. The process includes the collection of raw data of data preprocessing, model space establishment, parallel modeling of the orebody model and the visualization of orebody parallel body.

Firstly, the data preprocessing mainly carries out the quantification and the regular processing to the raw data, unifies the data dimension, filters out the invalid data, and through the rule processing reduces the storage data scale.

Secondly, the model space establishes the coordinate system for the body mineralization model, and the preprocessing data is mapped into the body mineralization model coordinate systems through the steps of coordinate transformation (translation, transformation, and rotation transformation), data discretization and spatial mapping. 
Then, the parallel computation of the orebody model is divided into two parts: the parallel computation of the body and the orebody modeling. The parallel computation of the body is used to compute the grade information of each body by using the parallel space estimation algorithm for the body mineralization model, and the orebody modeling is the establishment of the Orebody model by using the set operation.

Finally, a three-dimensional orebody model is drawn by using the parallel volume visualization technique to achieve the simulation results. In the process of drawing the body mineralization model, it is found that the algorithm has a significant dependence on the graphics card in parallel computation. Because of the video card's memory limit, the body data of the orebody model cannot be loaded into the video card at once, but the original model must be decomposed into several sub-models and then processed in batches.

Processing involves adding a need for serial operation, so that the speed is affected. In addition, in the threedimensional parallel volume rendering algorithm of the mineralization model, there is still a large number of orebody (inactive Volume pixel) that is not involved in drawing, especially in sparse-body data. To solve this problem, a linear Octree index is established for the parallel-body mineralization model, which can greatly reduce the time of the space element to the GPU-side sampling process.

\subsection{Algorithm Optimization}

At the same time, before pixel sampling, it is better to optimize the optical volume rendering algorithm by using the bounding box to select and remove voxel points, and the algorithm flow of the child threads is shown in Figure 3.

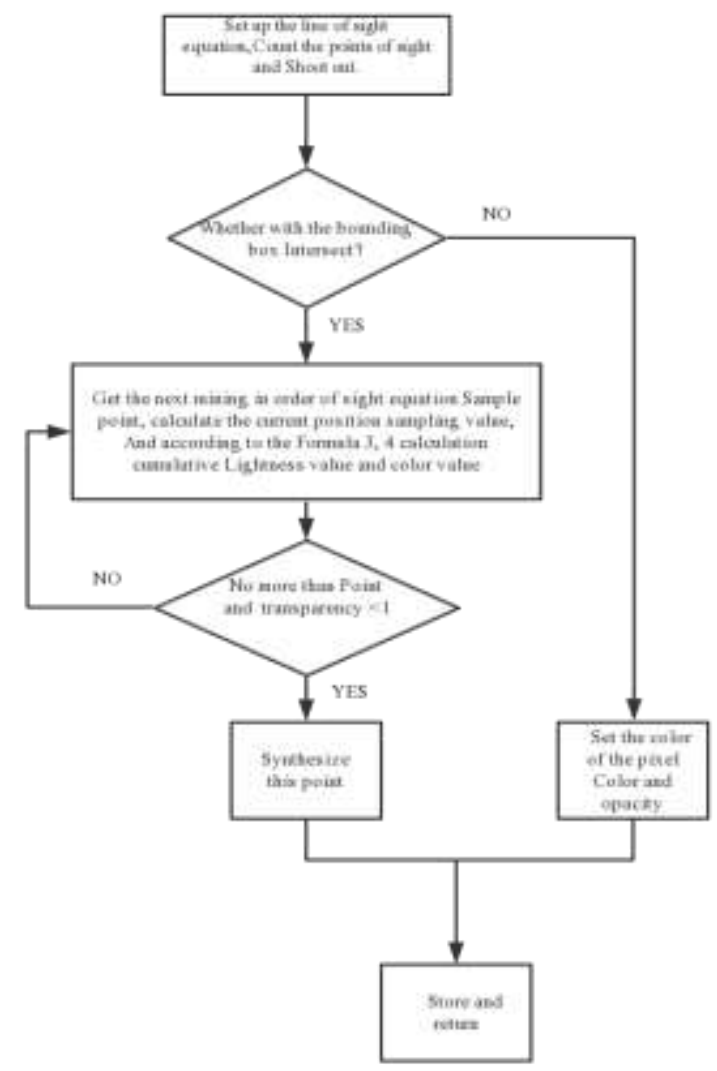

Figure 3. Volume rendering algorithm optimization flowchart

\section{Experimental Verification}

Taking an actual geologic data of a mine as an example, the stability, acceleration ratio and data accuracy of the algorithm are tested. Due to the large orebody data of the iron mine, the system is divided into a 3-block model according to the card processing capability, and the result of the parallel interpolation calculation is as shown in Table 1. 
Table 1. Comparison of drawing speed of three-dimensional parallel ray projection (sec)

\begin{tabular}{|c|c|c|c|c|}
\hline Project & Size of mine model & Serial drawing time & Parallel drawing time & Acceleration ratio \\
\hline Experiment 1 & $256 \times 256 \times 71$ & 0.697 & 0.183 & 3.81 \\
\hline Experiment 2 & $500 \times 500 \times 71$ & 2.846 & 0.754 & 4.77 \\
\hline Experiment 3 & $3700 \times 4237 \times 71$ & 159.458 & 53.067 & 4.01 \\
\hline
\end{tabular}

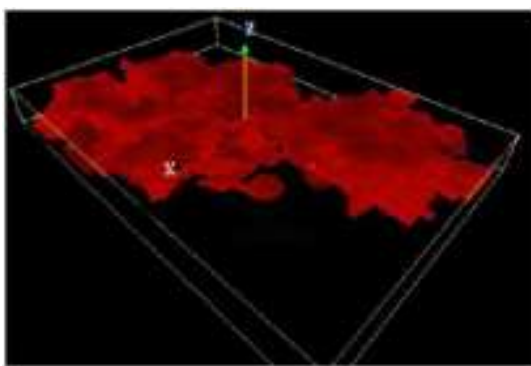

(a) Iron ore

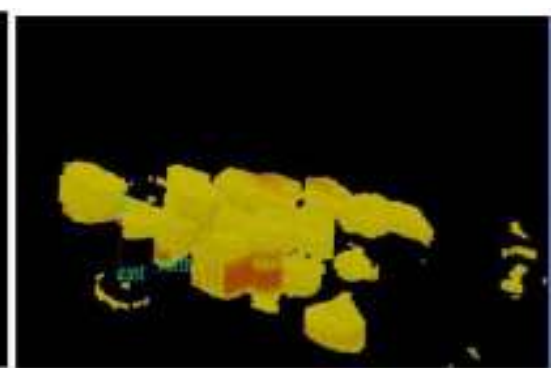

(b) An underground gold ore

Figure 4. (a) Iron ore (-288m -358m); (b) An underground gold ore body drawing of the parallel body of the three-dimensional orebody

The experimental results show that when the size of the model is too large and the graphics card cannot meet the required resources, the acceleration ratio will decrease greatly but still have a certain acceleration function.

Acceleration ratio is an important measure of parallel algorithms. For the specified parallel computing system, the best case of the serial algorithm execution time is a multiple of the parallel algorithm execution time, as shown in Equation (3).

$$
\text { Acceleration ratio }=\frac{\text { Execution time of the optimal serial algorithm }}{\text { execution time of the parallel program }}
$$

\section{Conclusions}

In this paper, a parallel-body mineralization model is defined based on the model of body mineralization, and the parallel computation theory is introduced. Secondly, based on the parallel body mineralization model, the parallel Ray projection method for the body-oriented mineralization model is designed, and related problems of parallel rendering algorithm are analyzed. A corresponding optimization method is put forward. Thirdly, the sampled GPU completes the multithreaded computation process and the pixel synthesis part of parallel body rendering. Finally, a parallel system prototype is established, and the actual data of mine enterprise is applied to obtain a better speedup. The correctness and reliability of the algorithm are verified. This lays a foundation for further application of parallel computing in mine field.

\section{Acknowledgements}

This study was financially supported by the National Natural Science Foundation of China (No. 51304078), Henan Provincial Education Department Project (No. 17A440003), North China University of Water Resources and Electric Power, Henan Provincial Key Research Projects for Colleges and Universities (No. 17A440003), and the Key Research Funding Scheme of Henan University (No. 18A520035).

\section{References}

1. M. Bin, "Research on Integrated Modeling and Visualization Simulation Technology of Mine Compound Field," Beijing University, 2009

2. F. Jun and F. X. Yang, “An Efficient Rendering Algorithm for Ray-casting,” Computer Technology and Development, Vol. 8, pp. 67-70, March 2014

3. M. Levoy, "Display of Surfaces from Volume Data," Computer Graphics and Applications, Vol. 3, pp. 29-37, August 1988

4. R. H. P. Harvey, "Ray-casting Architectures for Volume Visualization," IEEE Transactions on Visualization and Computer Graphics, Vol. 3, pp. 210- 223, May 1999

5. H. Nan and D. H. Wei, “An Efficient Rendering Optimization Algorithm for Ray Casting," Aerospace Medicine and Medical Engineering, Vol. 4, pp. 265-269, August 2015

6. S. Peng and G. B. Rong, "An Adaptive Beam Rendering Algorithm with Improved Intersection," Computer Engineering and Applications, Vol. 4, pp. 70-72, July 2008

7. N. C. Xia, F. Hui and D. H. Qiu, "A Fast Ray Casting Algorithm for Medical Volume Rendering," Journal of System Simulation, Vol. S1, pp. 343-346, August 2006 